\title{
LA ECOLOGÍA DE SABERES COMO IMPULSO TEÓRICO A PROYECTOS ECONÓMICOS REGIONALES. ESTUDIO DE CASO: LAS POTENCIALIDADES ECOTURÍSTICAS DE LA CIUDAD DE IPIALES, COLOMBIA
}

\section{THE ECOLOGIES OF KNOWLEDGES AS A THEORETICAL IMPULSE TO REGIONAL ECONOMIC PROJECTS. CASE STUDY: THE ECOTOURISM POTENTIALITIES OF IPIALES CITY, COLOMBIA}

\section{A ECOLOGIA DO CONHECIMENTO É IMPULSO TEÓRICO PARA PROJETOS ECONÔMICOS REGIONAIS. ESTUDO DE CASO: AS POTENCIALIDADES DO ECOTURISMO NO CIDADE DE IPIALES, COLOMBIA}

LATIF OLMOS_Soraya; NIÑO ARTEAGA_Yesid

Magíster (C) en Marketing Digital y Comercio electrónico, Universidad Internacional de la Rioja, España. Docente Departamento de Comercio Internacional y Mercadeo, Universidad de Nariño. Email: sorayalatiff@udenar.edu.co, Colombia.

Doctor (C) en Ciencias de la Educación, Universidad de Nariño-RUDECOLOMBIA. Docente de Formación Humanística, Universidad de Nariño. E-mail: yesidnio@ hotmail.com, Colombia.

Recibido: 3 de mayo de 2020

Aprobado: 12 de agosto de 2020

DOI: https://doi.org/10.22267/rtend.202102.146

\section{RESUMEN}

Este artículo de reflexión aspira a presentar una interpretación crítica de lo que puede significar la construcción de proyectos de emprendimiento económico en sectores vulnerables del municipio de Ipiales. El artículo se desprende de una investigación cualitativa en la que se pretende vincular a la población local con las potencialidades ecoturísticas en economías cambiantes, en entornos competitivos y devastadores del ecosistema natural, bajo la premisa de que se pueden buscar opciones 
que puedan equilibrar situaciones relacionadas con la depredación ambiental y con el mejoramiento de la calidad de vida de las personas que laboran en entornos rurales. De allí que el artículo aborde un contexto del ecoturismo desde la economía regional, luego se plantee una reflexión sobre el sistema económico desde la ecología de saberes y finalmente se configure el problema práctico y algunas sugerencias dentro del diseño y la consolidación de proyectos de impacto regional. Se llega a la conclusión de que es muy importante permitir la participación comunitaria en lugar de la apropiación del ecosistema por parte de la racionalidad capitalista.

Palabras clave: ecoturismo; desarrollo humano alternativo; ecología de saberes; diversidad epistemológica; pensamiento alternativo.

JEL: O15; Q01; Q27; Q57; R11

\begin{abstract}
This reflection article want to present a critical interpretation about the meaning of construction of economic entrepreneurship projects in vulnerable sectors of the municipality of Ipiales. The article develops from a qualitative research in which it is intended to attach at local population with the ecotourism potentialities in changing economies, in competitive and devastating environments of the natural ecosystem, under the premise that options can be found that can balance situations related to environmental depredation and with the improvement of the quality of life of people who work in rural settings. Thence, this article shows a context of ecotourism from the regional economy, then make a reflection about the economic system from the ecologies of knowledges and finally the practical problem and some suggestes within the design and consolidation of projects that seek community participation instead of the appropriation of the ecosystem by the capitalist rationality.
\end{abstract}

Keywords: ecotourism; alternative human development; ecologies of knowledges; epistemological diversity; alternative thinking.

JEL: O15; Q01; Q27; Q57; R11

\title{
RESUMO
}

Este artigo de reflexão tem como objetivo apresentar uma interpretação crítica do que a construção de projetos de empreendedorismo econômico pode significar em setores vulneráveis do município de 
Ipiales. $\mathrm{O}$ artigo emerge de uma pesquisa qualitativa na qual se pretende vincular a população local às potencialidades do ecoturismo em economias em mudança, em ambientes competitivos e devastadores do ecossistema natural, sob a premissa de que podem ser encontradas opções que possam equilibrar situações relacionadas a depredação ambiental e com a melhoria da qualidade de vida das pessoas que trabalham no meio rural. Nesta ordem de ideias o artigo mostra um contexto de ecoturismo da economia regional, em seguida é proposta uma reflexão sobre o sistema econômico a partir da ecologia do conhecimento e, finalmente, o problema prático é configurado na concepção e consolidação de projetos que buscam a participação da comunidade em lugar da apropriação do ecossistema pela racionalidade capitalista.

Palavras-chave: ecoturismo; desenvolvimento humano alternativo; ecologia do conhecimento; diversidade epistemológica; pensamento alternativo.

JEL: O15; Q01; Q27; Q57; R11

En memoria del maestro Luis Carlos Gómez Jiménez

\section{INTRODUCCIÓN}

La vinculación cultura/economía tiene un profundo impacto en las relaciones comerciales y en la construcción del sentido de lo económico. Este es el caso del municipio de Ipiales, Colombia, donde elementos como la cosmogonía indígena, el mestizaje, el sincretismo religioso, pero también la globalización y racionalidad capitalista repercuten no sólo como aporte a la economía y a la cultura del país, sino como un espacio propicio para explorar teorías que puedan ampliar la reflexión, así como la participación de la identidad latinoamericana en la construcción de esa realidad económica. Esto se apoya en la concepción de Boaventura de Sousa Santos $(2014 ; 2017)$ en la que Latinoamérica conforma un espacio donde puede afirmarse la diversidad epistemológica, la pluralidad de conocimientos, la multidimensionalidad humana y la renovación de los discursos. Una "ecología de saberes" que busca la objetividad analítica, pero al mismo tiempo el desarrollo de la dimensión éticopolítica que considera que también existe una crisis cognitiva global (Santos, 2014). 
Aunque dentro de esta hibridación cultural que caracteriza el territorio latinoamericano, existen factores que han impedido que se desarrolle ese pensamiento que emerge desde las mismas luchas étnicas y sociales en Latinoamérica. Traducido a las relaciones comerciales es claro el ejercicio de una cultura de poca asociatividad, la primacía del consumismo, la acumulación de capital en unos pocos no permite que microempresas o proyectos de emprendimiento enfocados en el desarrollo social y comunitario puedan crecer o desarrollarse. Esto intensifica que en regiones como el municipio de Ipiales se tienda a desarrollar el trabajo independiente e ilegal asociado al contrabando (Ministerio de Justicia de Colombia, 2016; Defensoría del Pueblo de Colombia, 2017), además incide en que los proyectos de vida laboral de muchas familias se vean delimitados a las ventas diarias para el sustento familiar e individual (Organización Internacional para las Migraciones, 2015).

En cuanto al ecoturismo, este es un tema relativamente nuevo para el municipio de Ipiales, aunque se han desarrollado proyectos esporádicos dentro del sector privado, las investigaciones en este tema y la vinculación de la comunidad en este tipo de actividades casi no se han desarrollado. En Colombia las investigaciones se centran en desarrollar estrategias ecoturísticas en la Amazonía, el eje cafetero, Magdalena, Guajira, Huila y Cundinamarca (Ospina, Mora y Romero, 2013). En Nariño se tienen investigaciones como la de Lagos y Riascos (2007) quienes proponen al ecoturismo como una alternativa de desarrollo regional en cuanto a la conformación de una empresa de economía mixta. García y Triana (2007) quienes pretenden implementar una propuesta ecoturística al determinar el potencial ecoturístico del Páramo de la Paja Blanca en Pupiales. Las características geográficas y naturales del departamento de Nariño permiten una alta biodiversidad asociada con la gran riqueza cultural y biológica de sus ecosistemas, diversidad biológica que es resultado de la compleja variedad de pisos térmicos y la presencia de costas y la cordillera de los Andes (Montenegro-Muñóz, Delgado, Pantoja, Calderon-Leyton y Noguera-Urbano, 2019).

Cabe mencionar que lo descrito en el presente artículo de reflexión continúa la invitación de seguir desarrollando investigaciones cuyo eje central sea el ecoturismo, que fue expresada dentro del Trabajo de Grado "Estudio de la oferta ecoturística de Ipiales desde una perspectiva de desarrollo socioeconómico alternativo" realizado en el año 2016, el cual tuvo como objetivo indagar en el potencial ecoturístico de Ipiales, su relación con la cultura y la posibilidad de generar comercio responsable. Para eso se profundizó y continuó con el enfoque educativo, alternativo y social que se 
propuso, toda vez que se tiene la voz y el pensamiento de la comunidad como la base o elemento requerido para establecer proyectos económicos de este tipo. Se continuó con el trabajo de sensibilización, formación y diálogo con una familia rural de cinco integrantes que voluntariamente deseó participar de esta experiencia. Se profundizó en el establecimiento de tratos y diálogos con propuestas teóricas tejidas en Latinoamérica, como la ecología de saberes, bajo el presupuesto de que puede conformar un "impulso teórico" (Lledó, 2015) que permita ampliar la conciencia crítica, la sensibilización ecológica, mejorar la inteligencia económica de las familias, afirmar la voluntad de transformación, así como la posible construcción de otros sentidos en la realidad social y en la teoría económica.

Para eso se emplea la experiencia escritural del ensayo y la fundamentación teórica en el enfoque de los estudios culturales como garantía de diálogo en el plano racional y así dar lugar a las inquietudes y voces de los participantes en las reflexiones del proceso de escritura de este artículo. Como descripción del problema se tiene que existe un gran desconocimiento de las potencialidades ecoturísticas de Ipiales, asimismo del aprovechamiento de los saberes de las comunidades, por tanto, se cree erróneamente que es una frontera con pocos atractivos ecoturísticos y que debe asimilar las imposiciones de una clase capitalista dominante (Grosfoguel, 2006).

Frente a esto último se plantea el objetivo de participar en la ampliación de la reflexión sobre las condiciones para incursionar en el mercado ecoturístico desde una perspectiva de desarrollo humano alternativo contenida en la concepción teórica de la ecología de saberes (Santos, 2017; 2014; Leff, 2011), para que así se pueda contribuir mínimamente a un posible cambio de racionalidad en las relaciones humanas y comerciales, teniendo en cuenta a su vez la realidad socioeconómica de familias que aún no logran consolidar un proyecto laboral exitoso, o por lo menos, duradero, que dentro de su vida cotidiana comparten el la idea de dar reconocimiento a un pensamiento relacionado con lo ecológico y lo axiológico.

\section{REFERENTES TEÓRICOS}

Los estudios culturales permiten trazar reflexiones respecto a los impactos negativos a la vida social y a los saberes tradicionales o populares que la colonialidad y el capitalismo han implementado dentro 
de su modelo de desarrollo económico (Grosfoguel, 2006). Incluso el conocimiento científico y económico no ha quedado exento de la influencia negativa del capitalismo (Santos, 2019), de allí que el enfoque de los estudios culturales permita trazar alternativas ético/epistémicas en oposición a epistemologías eurocéntricas de corte capitalista, colonialista y patriarcal (Grosfoguel, 2006). Dentro de este enfoque, la ecología de saberes de Boaventura de Sousa Santos (2014; 2017) anuncia su posibilidad de crítica, la cual consiste en emancipar a la razón y al sentir humano de la instrumentalización y la hegemonía del mercado. Para eso establece una confrontación intelectual y un distanciamiento de un tipo de racionalidad que asume posturas opresoras e indolentes las cuales niegan el diálogo, la participación o el debate ético con otras expresiones de los saberes humanos.

Para este pensador hay una relación ineludible entre injusticia social e injusticia cognitiva (Santos, 2014; 2017). Argumenta que se ha monopolizado una sola clase de saber a la vez que se reduce y rechaza otros tipos de saberes, estas formas de separación y rechazo de saberes o "líneas abismales" (Santos, 2014) establecen dicotomías que resultan peligrosas tanto para la creación de conocimiento como para un posible bienestar social e intercultural. El autor propone entonces su concepto de ecología de saberes como una forma de asumir la pluralidad de las prácticas científicas y de valorar la interdependencia y posibilidades de saberes no científicos (Santos, 2017).

La ecología de saberes se puede entender como acciones del "encuentro mutuo y del diálogo recíproco que sustenta la fertilización y la transformación reciprocas entre saberes, culturas y prácticas que luchan contra la opresión" (Santos, 2019: 346). Esta propuesta tiene relación con el concepto de diálogo de saberes que es profundizado por Leff (2011) como crítica a la racionalidad capitalista, pero al mismo tiempo, como búsqueda creativa para proponer una integración entre los distintos saberes con su naturaleza ética, filosófica, ecológica.

Tanto Leff (2011) como Santos (2019) distinguen la hegemonía de un saber dominante que tiende a imponerse, desde la racionalidad capitalista e indolente, por encima de otros saberes que, en su mayoría son alternativos, cotidianos, populares o que parten de cosmovisiones y tradiciones ancestrales. La propuesta tanto del dialogo de saberes como de la ecología de saberes gira en proponer un tejido de saberes que pueda articular tanto la profunda relación entre el ser y el saber de las cosmovisiones como la apertura e inventiva propia de las ciencias, en palabras de Leff, "un dialogo 
de saberes en donde se confrontan diversas racionalidades y tradiciones" (Leff, 2011: 21). Carrasco (2020) menciona que el diálogo de saberes permite trazar un marco para la superación de la dicotomía entre naturaleza y cultura, sin embargo, resalta que esto solo inicia con la participación de los sistemas de conocimiento y prácticas locales en el ejercicio científico. Lo que sustenta el reconocimiento de un principio integrador entre naturaleza y sociedad que a su vez permite advertir la interconexión de intereses e intenciones que se entrecruzan con los sentidos de la desigualdad económica y la diversidad cultural de un territorio.

Confrontación intelectual, rechazo al capitalismo, diálogo, relación y diversidad entre saberes, participación de las comunidades, inclusión de los diferentes contextos, mostrar la historia de la relación entre los distintos saberes, intensificar la actitud crítica y la creación de acciones contra la opresión, un fondo ético-político e intercultural (Santos, 2017), así como la apertura hacia otra racionalidad social, hacia nuevas "verdades por venir", serían características de esta ecología de saberes.

La ecología de saberes se fundamenta en que el conocimiento humano principalmente es interconocimiento, recurre a afirmar tanto la diversidad epistemológica del mundo (Santos, 2019) como la compatibilidad entre los valores cognitivos y los ético-políticos (Santos 2017; Leff, 2011). La pregunta entonces sería: ¿cómo lograr una eficiente intervención humana que entrelace la condición educativa, ecológica y cultural, para llegar a la afirmación económica e iniciar una gestión acerca de la problemática ecológica que se presenta en la actualidad? ¿Es posible pensar el ecoturismo en relación con la ecología de saberes? ¿Cómo vincular a la comunidad a una posible reinterpretación de los problemas económicos dando lugar a la diversidad y a la dimensión ambiental del pensamiento?

Cabe destacar el crecimiento a nivel mundial de los proyectos ecoturísticos que se han consolidado como una posibilidad de ampliación de la actividad comercial de ciertas regiones latinoamericanas (Rodríguez, 2019), pero a su vez cabe resaltar que pocos son los que consideran que ese desarrollo integral beneficie al corto y largo plazo a las comunidades locales que participan de estos proyectos. Por ejemplo, esto se evidencia en la actual disputa entre cierta parte del sector privado hotelero y las comunidades indígenas y campesinas que habitan en la Sierra Nevada de Santa Marta. Al parecer se 
han establecido violencias directas sobre estas comunidades a la vez que se empieza a desarrollar estos proyectos que no solo deterioran el ecosistema, sino que solo consideran como algo "exótico" el pensamiento de comunidades indígenas llegando así a su depreciación.

Frente a este debilitamiento de la razón por el influjo capitalista, la realidad política de un país como Colombia ha sido el escenario propicio para que se desarrollaran situaciones propias de un conflicto armado y las consecuencias que este conflicto genera: depredación de los recursos naturales (renovables y no renovables), altos índices de analfabetismo, morbilidad, mortalidad con la consecuente baja de la calidad de la vida poblacional y el desarrollo de una economía ineficaz donde prima una fuerte desigualdad. En este punto Trouillot sostiene:

La historia reciente del mundo está caracterizada por una serie de cambios fundamentales en la espacialización. Los cambios en la espacialización de mercados- el mercado de capital (financiero e industrial), el mercado de trabajo y el mercado de bienes de consumo- crean espacialidades yuxtapuestas que no están sincronizadas pero que, juntas, contribuyen a dar su forma actual a la economía mundial. Segundo, y tan importante, el dominio del capital financiero sobre los tipos y volúmenes de flujos globales ahora establece las principales direcciones y tendencias de la economía mundial. Tercero, entre estas tendencias predomina la desigualdad creciente entre y a través de las fronteras políticas (Trouillot, 2011: 105).

En este sentido, la situación actual de un municipio como Ipiales se puede ver afectada por la depredación propiciada por conductas socioculturales, por tanto, económicas, que degradan no sólo el aspecto comercial del municipio, sino también, los lugares que pueden considerarse como espacios ambientales. Esta espacialización capitalista genera destrucción de los recursos naturales, donde se busca afirmar la peripecia convencional de incrementar la producción como respuesta a la problemática económica regional.

Desde otro punto de vista, lo que sugiere la ecología de saberes es dejar de sostener una racionalidad basada en el reconocimiento de los saberes eurocéntricos, con ese desplazamiento se puede observar cómo es de importante considerar el pensamiento de las comunidades locales dentro de proyectos de impacto regional. Al contrario del ejemplo de la Sierra Nevada de Santa Marta, el ecoturismo puede 
ser visto como fuente de desarrollo regional o comunitario, y no solo empresarial, que puede brindar a las comunidades, mediante la relación responsable con el contexto natural y el fortalecimiento del saber cultural, oportunidades de trabajo digno, mejoramiento de sus servicios básicos y mejores oportunidades de formación, de convivencia, de trabajo en valores, siendo el ecoturismo un componente que puede sugerirse como estrategia para afianzar un posible desarrollo económico alternativo.

Es así como la intención de darle impulso teórico distinto u horizonte de voluntad e inteligencia diferencial a los proyectos que se afiancen en la región permite que la reflexión teórica identifique otras perspectivas de conocimiento dentro de su inmanencia consolidada en la práctica (Lledó, 2015).

De allí que la ecología de saberes permita observar en las disciplinas de las ciencias sociales y económicas que la idea de desarrollo no solamente pueda apoyarse o sustentarse en la "lógica de la monocultura del conocimiento y del rigor científico", esto no quiere decir que se oponga a una experiencia del conocimiento científico, sino al contrario, permite ampliar y pluralizar esta experiencia con "otros saberes y criterios de rigor y validez que operan de forma creíble en prácticas sociales que la razón metonímica declara no existentes” (Santos, 2017: 237).

En este orden de ideas, el desarrollo ecológico del ecoturismo presenta ciertas dimensiones que se tendrían que concretarse. El desarrollo del ecoturismo puede centrarse en la relación con la naturaleza, la cultura y la conservación de los sistemas ecológicos. El turista tiene que comprometerse a estudiar las características del paisaje sin deteriorarlo, contaminarlo o dañarlo, es decir, provocar la toma de conciencia frente a las consecuencias irreversibles del daño a los ecosistemas. El proceso de conservación depende de los cobros a los turistas destinados a la conservación ambiental y el desarrollo económico de comunidades locales (Björk, 2000).

Esto indica que es posible lograr una perspectiva formativa del ecoturismo. Los conceptos de desarrollo humano, desarrollo comunitario, implican que el ser humano critique los efectos y consecuencias del consumismo y el desarrollo industrial. Ahora bien, esta crítica puede estar presente en todo proyecto ecoturístico para que las actividades proporcionen mejores relaciones con el 
ambiente o fomenten una cultura de respeto y valores enfocados a la ecología. También se puede orientar esta consolidación de proyectos hacia un des-aceleramiento del crecimiento económico ya que la innovación a su vez puede construirse por fuera de la acumulación de capital (Max-Neef y Smith, 2011). Esto quiere decir que el desarrollo humano está relacionado con el bienestar social e individual donde se reconozcan como elementos de su estar en el mundo: el cuidado-protección del medio ambiente, la existencia de otras comunidades y su propia identidad cultural.

Los procesos de producción dentro de una racionalidad capitalista han establecido métodos económicos y comerciales donde el medio ambiente ha sido vinculado como mero objeto destinado a explotarse desde la "ceguera del mercado". Según Leff (2004) al asumir los principios de una ciencia económica basada en una racionalidad desligada del sentido de lo ecológico en sus formas de producción y que es determinada "por las leyes ciegas del mercado", se asume un propósito sintético sostenido en el potencial tecnológico que se impone al componente vital de lo ecológico. En este orden de ideas, no es una exageración encontrar que las relaciones humanas y las relaciones con los saberes se presenten en la actualidad como dependientes a los criterios de explotación y acumulación de capital, a la crisis económicas y políticas impuestas sobre lo humano, caracterizándose por el desinterés por lo ecológico y que consiente una alienación caracterizada por la emocionalidad regulada por lo tecnológico, el modelo de lo hegemónico y la vaciedad crítica (Santos, 2017).

La ecología de saberes propuesta por Boaventura de Sousa Santos indica que la producción de conocimiento puede estar vinculada a una intervención intersubjetiva e intercultural con la realidad. La ecología de saberes invita a un cambio de perspectiva entre la relación humanidad/mundo de la vida e invita a reconocer en la producción de conocimiento la disrupción de lo popular en relación con lo subalterno y lo alternativo como una experiencia de resistencia de lo local frente a las regulaciones e invisibilización del mercado capitalista global.

Para Boaventura de Sousa Santos (2014) el modo de ser, pensar y estar de los seres humanos debería salir de esa "epistemología de la ceguera" y de esas "líneas abismales" que generalmente se usan y transmiten en el discurso económico asentando la desigualdad a través de dicotomías que permean tanto las subjetividades como las relaciones humanas: culto-inculto, conocimiento-ignorancia, 
seguridad-criminalidad, competente-incompetente, clase alta-clase baja, productivo-estéril, apto-no apto, desarrollado-no desarrollado.

Una ecología de saberes subsiste en la enseñanza de dinámicas de emancipación que principalmente tienden a proveer resistencias frente a los movimientos de regulación del pensamiento crítico en entornos no académicos. La idea de establecer resistencias enfatiza de manera directa en el cambio de mentalidad que se consuma desde la adopción de una racionalidad basada en la lógica capitalista. Por eso se considera pertinente establecer una constante problematización ante la relación que subyace entre el sentido de lo humano y el peso de la racionalidad capitalista (Niño, 2019). De esta manera si se problematiza el sentido de lo humano en los proyectos que tienden a crear trabajo o mejoramiento económico, así como en los conceptos de bienestar regional y desarrollo, también puede diseñarse, plantearse y posibilitarse de manera crítico-alternativa, otras formas de impulso teórico (Lledó, 2015) dentro del pensamiento económico que quizás pueden resultar relevantes en el contexto del departamento de Nariño.

\section{MÉTODOLOGÍA}

El tipo de investigación fue cualitativo y se enmarcó en el enfoque teórico de los Estudios Culturales. Como método de trabajo se optó por implementar una etnografía reflexiva de corte crítico social (Apud, 2013) que afirma su traza desde la reflexividad que provoca la comprensión de los fenómenos a través de la profundización en la perspectiva de los participantes directos de los fenómenos culturales en sus contextos naturales.

En este caso de una familia de cinco integrantes del sector rural del municipio de Ipiales que no posee una forma de trabajo formal y se dedica a funciones varias de comercio independiente. Se pretende desarrollar un ejercicio de ensayo académico a partir de la reflexividad como herramienta metodológica (Apud, 2013), que tuvo en su experiencia técnicas específicas en la recolección de información: las entrevistas a profundidad y el taller educativo. Con estas técnicas se buscó hacer un diálogo, más que una indagación, respecto a los hechos o situaciones con los cuales se perciben en relación con el problema del desconocimiento de potencialidades ecoturísticas. A su vez se buscó 
establecer un acercamiento al concepto de ecología de saberes y así reflexionar en conjunto sobre el impacto del capitalismo en la mentalidad de personas que viven en contextos rurales vulnerables.

La escritura del trabajo a manera de ensayo se desarrolla para transmitir la reflexividad desde las comprensiones teóricas y prácticas frente al interrogante: ¿cómo construir sentidos de reflexión intersubjetivos frente al problema económico que atraviesan las familias del sector rural, que no poseen un trabajo estable o subsisten de manera informal?

El estudio presupone un ejercicio de constante reflexividad para llegar a situaciones concretas invisibilizadas, explorar y describir para luego generar perspectivas teóricas que puedan usarse como catalizador de un pensamiento crítico que indague en la dimensión de lo humano (Niño, 2019). Por eso se procura describir el desarrollo del texto y la búsqueda de alternativas que se puedan inducir desde el sentir y el pensar de los participantes respecto a la realidad del problema. La recolección de datos está influida por las prioridades dialógicas de los participantes más que por la aplicación de un instrumento de medición. Se concibe la producción de conocimiento desde una manera crítica donde prima la intersubjetividad.

La investigación se basó teóricamente en datos recopilados y en información transcrita desde otras fuentes de recolección, como libros especializados y revistas científicas, principalmente. Los datos primarios y la fuente de información primaria de esta investigación se obtuvieron con base en relatos orales, diálogos y testimonios que fueron recogidos en el proceso de investigación de cuatro meses, ejecutado en dos sesiones de dos horas por mes, con cuatro de los cinco miembros mayores de edad de una familia de escasos recursos, con estudios primarios y sin formación profesional y/o técnica, población perteneciente al área rural y que conocen muy bien los posibles lugares que puedan ser vistos con potencial ecoturístico.

La información se recopiló a partir de técnicas seleccionadas intencionalmente como la entrevista a profundidad y el trabajo teórico-práctico dentro de un taller educativo, que sirvieron para captar y relacionarse con la información y vivencias que puedan ser útiles en sucesos o acontecimientos de reflexividad. Los ciclos de la investigación fueron diagnóstico, reflexión, entrevistas, reflexión, trabajo grupal, entrevistas, reflexiones finales. La información recopilada de las fuentes secundarias 
permitió el respectivo análisis e interpretación de los testimonios apoyados en la triangulación bibliográfica con referentes de los estudios culturales y el pensamiento latinoamericano. Con el análisis de las entrevistas se buscó identificar algunos de los problemas que los miembros de una familia rural puedan tener al momento de formar un proyecto comercial o alguna alternativa de trabajo diferente a los tradicionales. A su vez se buscó que se generen posibles alternativas en tanto relaciones humanas y sociales para que así sea viable integrar espacios de trabajo pedagógico donde el desarrollo personal sea un medio para lograr un beneficio sostenible que a su vez logre transmitir expresiones culturales

En los diálogos, conversaciones y caminatas que se daban en las entrevistas se expresaban pequeñas discusiones sobre el posible potencial económico de la región y si ellos como familia y comunidad podían acceder a otras formas de desarrollo social o económico. La invitación a mantener una postura abierta al aprendizaje, pero a su vez una actitud crítica frente a la opresión, tal como lo señala la ecología de saberes (Santos, 2014) fue propicia para establecer la discusión sobre las posibles prioridades en un proyecto ecoturístico.

En los talleres se construyó en conjunto la base del análisis crítico: "es necesario proteger a las especies, paisajes, los ríos y que se participe de mejorar el medioambiente”. En este punto la voz de los participantes resume lo expuesto por Björk (2000), debido a que los elementos de desarrollo de estrategias y/o proyectos ecoturísticos tienen que incluir variables ecológicas, sociales y culturales, principalmente. En los que se pueda articular el manejo de los recursos de manera equilibrada y así no generar impactos negativos (ecológicos, económicos) en las regiones visitadas o que sirven del disfrute responsable del turista.

El taller educativo arrojó que la población rural no tiene conocimiento acerca de planes de desarrollo ecoturístico, existe una gran desconfianza frente a las acciones realizadas por entidades de carácter público y privado que puedan presentar proyectos de conservación o protección medioambiental. A su vez el trabajo grupal proyectó que resulta imperioso articular a las entidades públicas y privadas en un posible programa de conservación medioambiental y/o de emprendimiento ecoturístico, sobre todo 
darle relevancia a lo correspondiente a planes educativos y de trabajo formativo antes de iniciar proyectos que puedan llegar a tener impacto en las comunidades.

\section{DISCUSIÓN}

\section{Relevancia de las características socioculturales en proyectos de ecoturismo}

En la frontera colombo-ecuatoriana, específicamente en el municipio de Ipiales, el problema económico se acentúa por el aumento de la condición de vulnerabilidad, por el crecimiento de la explotación sexual y laboral de niños, niñas y adolescentes (Aldeas Infantiles SOS Colombia, 2017), por la constante violencia directa, de género e intrafamiliar (Defensoría del Pueblo de Colombia, 2017), los altos índices de suicidio (Ojeda y Villalobos, 2011), pero también por la violencia militarizada ejercida por la reorganización de grupos criminales y grupos pos-desmovilización (Ministerio de Justicia, 2016). A su vez el problema tiende a agudizarse por el complejo fenómeno de la migración ilegal y el desplazamiento forzado (Ruano, 2012), la delincuencia internacional y la trata de personas que también serían problemáticas que parecen tocar profundamente a la población, y por ende, a la economía de este municipio (Organización Internacional para las Migraciones, 2016).

Ipiales es considerado municipio agrícola en el que predominan relaciones comerciales propias de una frontera debido al paso de mercancías desde y hacia el Ecuador. Posee economías de subsistencia,

siendo los minifundios el medio para lograr la producción en el sector primario de la economía (pecuario, agrícola, forestal y pesca) supliendo así algunas necesidades de consumo básico. En su mayoría, son los agricultores quienes tienen que asumir gastos de producción y comercialización, lo que disminuye el potencial y afecta la producción. No hay acceso a procesos tecnológicos por el alto costo de las maquinarias agrícolas que difícilmente pueden ser conseguidas por los agricultores, además la falta de recursos y la poca inversión estatal ha impedido que la economía local se desarrolle. La tradición comercial en Ipiales ha sido eminentemente agropecuaria y comercial, aunque actualmente prolifera "lo informal", los negocios "independientes" o establecimientos temporales.

Aunque se debe considerar al mismo tiempo que en Ipiales se manifiesta el poco sentido de pertenencia por la identidad regional ya que se buscan estilos de vida que estén de acuerdo a la "modernización" o "desarrollo" de la lógica del mercado capitalista. Esta situación afecta las 
condiciones laborales y formas de emprendimiento alternativo en sus posibles proyectos económicos que se refleja en los altos índices de crimen y en la poca gestión ambiental en los habitantes del municipio, quienes no cuidan voluntariamente los espacios naturales, lo que provoca una falta de compromiso en el cuidado de los recursos no renovables ya existentes.

De ahí la importancia de articular las dinámicas propias de un desarrollo humano alternativo (dinámica social, dinámica económica, dinámica ecológica) que pueda dar apertura a un cambio de mentalidad en las relaciones económicas, sociales y medio ambientales. La primera relacionada con variables como el empleo digno, el salario justo, la consecución de vivienda, el acceso a los servicios esenciales. La segunda corresponde a la participación en la toma de decisiones, la seguridad de la sociedad y no de lo empresarial, el desarrollo de los derechos humanos, el beneficio de una salud y una educación de calidad. En cuanto a lo medio ambiental, esta parte se relaciona con la calidad del agua, los procesos para contribuir en el mejoramiento de la contaminación del aire, en el manejo responsable de los residuos, así como la interacción razonable con los espacios protegidos y el fortalecimiento de la postura crítica que el ser humano puede asumir frente al daño que históricamente se le ha hecho al entorno natural.

Estas dimensiones permitieron comprender que los participantes reconocen tener gran apropiación de la visión cosmogónica que tiene relación con la cultura del sur de América, la figuración simbólica de la Pacha-Mama y la Virgen de las Lajas, en cuanto al Departamento de Nariño, que vislumbra una relación armónica donde la tierra y el medio ambiente implican relaciones de vida y otras formas de interpretar el conocimiento gestado en lo social. Esta identificación permitió establecer diálogos en los que los participantes y los investigadores ampliaran el compromiso de no poner en riesgo el entorno ni a otras especies nativas, que deberían ser conservadas para el aprovechamiento de los recursos, tan importantes para una región donde sus creencias están ligadas al lugar de trabajo, al entorno natural y al espacio vital de la cultura y la tradición regional.

La orientación formativa desde una ecología de saberes en aspectos del ecoturismo así como la apertura al conocimiento popular y/o tradicional de la población, permitió encontrar prácticas viables dentro de lo económico que podrían considerarse como actividades que pueden ampliar la acción del 
ecoturismo: gastronomía, juegos tradicionales, atención a turistas, guías turísticos, alojamiento, conocimiento y uso de medicinas naturales, que pueden amplificar las características de la oferta para aprovechar estas actividades como posible sustento familiar y social.

En este sentido, el aspecto cultural se plantea como una idea-fuerza que los habitantes del municipio refundan entre la visión ancestral que tienen del medio ambiente, además de mostrar a Ipiales como un lugar turístico y culturalmente delineado para la comunidad nacional e internacional, intenta afirmar la realización de mejores oportunidades económicas y sociales para las personas, además de buscar opciones viables de sostenimiento medioambiental, que es un factor que está siendo afectado por la visión de políticas y formas de vida neoliberales.

La integración económica de los sectores populares de la sociedad puede ser tenidos en cuenta dentro de un posible proyecto ecoturístico para que la misma población local resulte beneficiada. De ahí emana la posibilidad de crear conciencia crítica para aportar con los intereses reales que la sociedad requiere y no lo impuesto por el falso imaginario de la explotación de la naturaleza.

\section{Posibles potencialidades ecoturísticas de Ipiales}

Al tener en cuenta la participación de la comunidad y la intersubjetividad en los talleres realizados, se pudo determinar que, entre los lugares con posible potencial ecoturístico en la periferia de la ciudad de Ipiales, se encuentran: La Piedra de los monos-Cañón Rio Guáytara, Sector rural del Puente de Rumichaca, Puente Nuevo, Yaramal y sectores aledaños. Además, se puso en consideración que las principales actividades dentro del ecoturismo podrían ser las caminatas ecológicas, el senderismo, la interacción cultural con las comunidades ancestrales, el avistamiento de aves y el conocimiento de plantas medicinales. Las actividades enfocadas al agroturismo pueden ser una potencialidad dentro de lo económico si existieran proyectos comunitarios donde sus miembros puedan ofrecer servicios de alojamiento, alimentación, orientación turística, participación en labores rurales o agrarias, brindar descripciones y muestras de los cultivos, que serían apropiadas para prestar servicios a visitantes y turistas.

Piedra de los monos: A casi dos kilómetros de distancia, iniciando desde el Barrio el Charco, se puede llegar a un espacio ancestral de la cultura de los Pastos, conocida como La Piedra de los Monos que, 
según los relatos de las personas que participaron en los talleres, fue un centro ceremonial donde se realizaban rituales religiosos. Lo más llamativo de este sitio es que aún existen petroglifos plasmados con la sabiduría de estos pueblos. Este sitio ofrece una evidencia para el reconocimiento del valor histórico y cultural del saber ancestral indígena.

Alrededores del Puente de Rumichaca: Este lugar es apreciado por turistas que disfrutan de la vista hacia el cañón del río Guáytara. La frontera entre Colombia y Ecuador ofrece la posibilidad de observar el recorrido del río y su paso por el cañón mediante su reconocido caudal oscuro e intempestivo. Si se hace un descenso por un costado del puente o por otras partes cercanas, se llega a segmentos de la rivera que pueden ser disfrutados por muchos visitantes, caminatas y acampadas serían actividades para quienes deseen encontrar conexión con la naturaleza y la cultura.

Puente Nuevo: Se ubica a quince minutos de viaje en carro o a 45 minutos a pie partiendo del parque 20 de Julio en Ipiales, se encuentra al sur oriente del municipio, se llega en descenso hasta el cañón del río Guáytara y en la vía que conecta con las veredas aledañas del municipio y con Sucumbíos. Al lado del camino están los paisajes rurales que son un atractivo natural para visitantes y turistas, se aprecian las montañas donde en su mayoría se realizan labores relacionadas con la agricultura. En el recorrido se puede encontrar variedad de plantas aromáticas, cultivos de moras, zanahorias, papas, arveja y maíz.

Yaramal: este lugar ancestral se caracteriza por compartir el territorio con la selva de la Victoria en la frontera con el Putumayo y la provincia de Sucumbíos en Ecuador. Aquí habita el Oso de anteojos, la única especie de oso nativa de América del Sur; especies como venados, chihuacos y tigres de montaña. También existe gran variedad de flora, plantas como vicundos, frailejones, pino enano colombiano, sobresalen en su entorno. La comunidad del resguardo indígena de Yaramal, que conforman el pueblo de los Pastos, protege este territorio como parte vital del conocimiento ancestral. A media hora de recorrido por tierra se llega al pueblo de Yaramal, que se caracteriza por su clima frío y ambiente selvático; la comunidad que habita este lugar pertenece en su gran mayoría a la comunidad indígena de Los Pastos o son campesinos mestizos con un profundo sincretismo religioso. Siguiendo el recorrido, se llega a algunos destinos ecoturísticos de gran importancia, aquí se nombran algunos: 
El Salado: este lugar se caracteriza por su clima de Páramo, está ubicado aproximadamente a 3400 m.s.n.m., se le llama el Salado porque increíblemente tiene yacimientos de agua salada. En este sitio las caminatas ecológicas se aprovecharían para admirar su extraordinaria vegetación, que hace de este un lugar verdaderamente mágico, donde se puede apreciar de la belleza de la vida silvestre y de la biodiversidad de diversos ecosistemas.

Llano Grande: lugar donde existe variedad de especies nativas, entre estos el Oso de Anteojos y el frailejón. Aquí se hace viable realizar caminatas ecológicas, reconociendo el reto ecológico y pedagógico que implica su conservación. Este lugar tiene senderos ancestrales, hechos por las antiguas comunidades indígenas, que aún no han sido borrados y todavía que pueden usarse.

Cerro Negro: este lugar es adecuado para realizar caminatas con cierto nivel de dificultad, los paisajes están compuestos por frailejones, de los senderos emergen rastros volcánicos que lo convierten en una experiencia sublime: la subida hasta la cumbre del cerro dura 4 horas y desde su cima se puede ver a plenitud la selva del Putumayo y los nevados que rodean el territorio de los Pastos. El recorrido aparte de mostrar la biodiversidad de especies de árboles, vicundos y frailejones, especialmente, conserva en su entorno diversidad de aves endémicas y migratorias, lo que permite conectarse y disfrutar del entorno como una experiencia de aprendizaje desde y con la Madre Tierra.

\section{Análisis crítico frente a posibles propuestas ecoturísticas con población rural}

Para Leff (2004) una racionalidad ambiental conduce a la construcción de la sustentabilidad desde un proceso que vincula la teoría con la práctica:

La valorización de la complejidad ambiental implica transformar la actual métrica que reduce la diversidad ontológica y axiológica del mundo a los valores objetivos, cuantitativos y uniformes del mercado, a una teoría cualitativa de una economía sustentable dentro de un pluralismo epistemológico y axiológico capaz de expresar los antagonismos entre la realidad económica y la racionalidad ambiental (Leff, 2004: 36).

Resulta difícil comprender que los saberes culturales de familias rurales pueden también participar de la construcción de teorías y prácticas en pro de extender una cultura ecológica. Tal vez esto inicie en 
reconocer la responsabilidad social e individual sobre la naturaleza para dar un nuevo significado al concepto de "valor" y que este sea construido en base a las relaciones entre la naturaleza y la sociedad o establezca equilibrios en la constitución de identidades culturales y ecológicas desde los espacios de conocimiento subalterno.

Por eso en primer lugar un proyecto económico puede emanar una propuesta de aprendizaje de algo distinto, de algo ecológico, humano, mitológico, literario, poético, pero también científico y argumentativo, en cuanto a conocimientos e información exteriorizada a las familias o a las comunidades. Al establecer dinámicas de intercambio simbólico y espacios para la enseñanzaaprendizaje del ecoturismo desde un enfoque alternativo, se pudieron analizar los siguientes aspectos: la población de Ipiales dice conocer aspectos generales del ecoturismo, debido a la posición geográfica del municipio, algunas actividades relacionadas con el entorno ambiental han sido desarrolladas por los habitantes casi de manera privada, sin tener en cuenta las potencialidades ecoturísticas que el espacio geográfico de Ipiales posee. Pero a su vez se conoció que Ipiales no está preparado para atender este tipo de actividades, porque según los participantes de esta investigación: "desde hace unos dos años eso ya lo están haciendo empresas privadas de Pasto o de Cali, nosotros tenemos que dedicarnos a otras cosas". Lo que demuestra que generalmente el sector privado sí tiende a realizar actividades ecoturísticas, pero sin considerar a poblaciones aledañas o comunidades locales. Aunque el ecoturismo es una alternativa viable de desarrollo y una potencialidad socioeconómica, en Ipiales todavía se carece de conciencia ambiental, de asociación y de crítica económica para lograr que esta sea una actividad que pueda aprovecharse satisfactoriamente y pueda incidir en un posible mejoramiento la calidad de vida de la población.

Esto corresponde a lo mencionado por Leff (2004) como un síntoma de un orden social totalitario, opresivo y enajenante que afecta la condición del ser humano como ser simbólico y ser productivo a trastornarlo como un ser pasivo y consumidor. La racionalidad económica define la conducta de los actores sociales según las motivaciones del mercado, el poseedor del saber económico es el indicado a desarrollar un proyecto, la ganancia y la utilidad se destinan por fuera de la zona local a la vez que se busca deslegitimar la posibilidad de las comunidades locales de participar de la apropiación social de ese conocimiento. 
En este caso se tuvo como eje temático las posibilidades de emprendimiento desde un modelo de desarrollo humano alternativo, la apertura de un pensamiento económico en relación con la ecología de saberes. Se llegó a un interesante aporte, ya que la trasmisión de información en un lenguaje nocientífico, pero sí riguroso, que reconozca las diferencias en cuanto valor humano, así como el reconocimiento de la educación como agente de cambio social, se puede considerar relevante dentro de las situaciones vitales para que la población se concientice y a su vez establezca prácticas sobre la importancia de cuidar y cambiar los tratos con las fuentes de vida.

La idea de ecoturismo se comprende desde los habitantes a partir de la definición básica: el ecoturismo se fundamenta en actividades que permiten el contacto directo con el medio ambiente, pero a eso se le añade que también consiste en conectarse espiritualmente con la naturaleza, con el sentido de la tierra, con la Pacha-Mama, en apreciar los sonidos, gestos y colores del medio ambiente, en realizar actividades que contemplen la reflexión humana a partir de la apreciación de los recursos naturales: el aire, el agua, la tierra, la montaña.

Algunos factores de la dimensión social y cultural a tener en cuenta para implementar proyectos ecoturísticos en la región pueden ser:

- El Cambio de percepción cultural y el ejercicio de una racionalidad ecológica con respecto a la experiencia de los turistas.

- La Concientización y sensibilización del cuidado ambiental y la problemática ecológica.

- Las Alternativas de saber ante el manejo y provecho inadecuado de los recursos naturales.

- Las Alternativas de saber ante la desatinada y despreciativa valoración de la cultura ancestral.

- Las Alternativas de saber ante situaciones de violencia, vulneración de derechos humanos.

- El conjunto de respuestas y/o posibilidades de la comunidad ante la falta de apoyo gubernamental y monopolización de la economía.

Cabe resaltar que el desarrollo del ecoturismo depende en gran parte de la capacidad de organización de la comunidad, de la voluntad local y de la educación e interpretación del entorno natural y humano, es importante el fortalecimiento de la gestión empresarial sostenible en servicios de guía turística, 
mejoramiento de la infraestructura, servicios de alimentación, elaboración de artesanías para incentivar e involucrar a la estructura social de la comunidad.

\section{CONCLUSIONES}

Luego del intercambio de saberes con las personas que participaron de esta investigación, luego de conocer sus inquietudes y experiencias, de dialogar sobre las diferentes características de una ecología de saberes y el trabajo ecoturístico, se logró conocer que el ecoturismo ha tenido poca o nula relación con iniciativas de participación local, que para su implementación es preciso el desarrollo de la infraestructura física que pueda favorecer a los viajeros o turistas, además se precisa generar elementos que permitan ampliar la participación de las comunidades de sectores rurales en la relación recíproca con los saberes locales. Asimismo, se debería priorizar el aprendizaje y enseñanza sobre la vulnerabilidad de los ecosistemas, su cuidado, su conservación y sus amplitudes en el desarrollo de proyectos que involucren lo medioambiental.

Con respecto a bienes o servicios que pueden servir como fuente alterna de ingresos a las comunidades está la producción de artesanías, productos alimenticios, servicios de restaurantes, servicios de hospedaje, entre otras actividades, más afines al campo ecoturístico están: la observación de aves, recorridos ecológicos, ciclo montañismo, paseos a caballo, ciclo rutas, observación de plantas y especies nativas, que pueden ser realizadas por personas de la comunidad campesina e indígena, si estas comunidades desean participar de posibles proyectos ecoturísticos.

Esta participación tendría que incluir sectores del gobierno municipal, empresas privadas, ONG y realizadores de programas sociales, desde la academia y la comunidad local, interesados en participar en procesos de desarrollo, que no se comprometan solamente por los beneficios económicos, sino en el acontecimiento o consecución de impactos sociales positivos para lo ecológico, lo cultural, la salud, la educación, además de generar respeto y afirmación de los valores culturales y ambientales.

Según lo anterior, el ecoturismo puede considerarse como una alternativa viable de desarrollo que a su vez puede desarrollarse dentro del municipio de Ipiales, puesto que sí existen espacios propicios para actividades que se relacionen con el medio ambiente a su vez que existe el deseo como el cambio 
de mentalidad dentro de la comunidad para participar en la planeación y el desarrollo de posibles actividades ecoturísticas.

Al percibir la riqueza cultural y natural de la zona, el ecoturismo entendido por fuera de un racionalidad capitalista y visto desde una ecología de saberes, se puede concebir como parte de un modelo alternativo de desarrollo económico que puede trabajar a su vez por la conservación de los recursos ambientales, que busca amplificar la conciencia ecológica en los participantes de las actividades ecoturísticas, así como en los habitantes del lugar, en este caso en la comunidad campesina, debido a que, ellos son los trabajadores y habitantes de los espacios en los que todo proyecto ecoturístico alcanza en su perspectiva. Con esto se extiende la invitación a que se sigan desarrollando investigaciones que favorezcan la diversidad epistémica para así contribuir mínimamente al cambio de paradigma en las relaciones humanas y comerciales en pro de apoyar desde la academia a reflexionar y buscar posibles alternativas al impacto negativo que como seres humanos hemos venido imponiendo al entorno natural.

\section{REFERENCIAS}

(1) Aldeas infantiles SOS Colombia. (2017). Informe anual. Recuperado de https://www.aldeasinfantiles.org.co/getmedia/6c576001-d2b9-4d13-8039-80224cf77ca2/AldeasInfantiles-SOS-Colombia-Informe-Anual-2017.pdf

(2) Apud, I. (2013). Repensar el método etnográfico. Hacia una etnografía multitécnica, reflexiva y abierta al diálogo interdisciplinario. Antípoda, (16), 213-235.

(3) Björk, P. (2000). Ecotourism from conceptual perspective, an extended definition of a unique tourism form. International Journal of Tourism Research, 2(3), 189-202. doi: doi.org/10.1002/(SICI)15221970(200005/06)2:3<189::AID-JTR195>3.0.CO;2-T

(4) Carrasco, N. (2020). Miradas decoloniales, interculturales y ecología política en la gobernanza de territorios. Utopía y Praxis Latinoamericana, 25(88), 34-40. doi: 10.5281/zenodo.3633746

(5) Defensoría del Pueblo de Colombia. (2017). Informe defensorial sobre las zonas de frontera. Bogotá, Colombia: Opciones Gráficas. 
(6) García, G. y Triana, M. (2007). Ecoturismo en el páramo de la Paja banca, una estrategia de conservación del páramo y de desarrollo sostenible para los municipios de Pupiales, Iles, Gualmatán, Sapuyes, Guachucal y Ospina (Tesis de especialización). Universidad de Nariño, Pasto, Colombia.

(7) Grosfoguel, R. (2006). La descolonización de la economía política y los estudios postcoloniales: transmodernidad, pensamiento fronterizo y colonialidad global. Tabula Rasa, (4), 17-46.

(8) Lagos, E. y Riascos, D. (2007). El ecoturismo como alternativa de desarrollo regional integral: caso volcán Azufral (Tesis de especialización). Universidad de Nariño, Pasto, Colombia.

(9) Leff, E. (2004). Racionalidad ambiental. La reapropiación social de la naturaleza. México: Siglo XXI.

(10) Leff, E. (2011). Aventuras de la epistemología ambiental: de la articulación de las ciencias al diálogo de saberes. México: Siglo XXI.

(11) Lledó, E. (2015). La memoria del logos. Barcelona, España: Taurus.

(12) Max-Neef, M. y Smith, P. (2011). La Economía Desenmascarada. Del poder y la codicia a la compasión y el bien común. Barcelona, España: Icaria editorial.

(13) Ministerio de Justicia de Colombia. (2016). Caracterización regional de la problemática asociada a las drogas ilícitas en el departamento de Nariño. Bogotá, Colombia: Imprenta Nacional.

(14) Montenegro-Muñóz, S. A., Delgado, F., Pantoja, Y. P., Calderon-Leyton, J. J. y Noguera-Urbano, E. A. (2019). Especies emblemáticas para la conservación de ecosistemas en el departamento de Nariño, Colombia. Ecosistemas, 28(3), 174-184. doi.: 10.7818/ECOS.1750

(15) Niño, Y. (2019). Problematizar lo humano en educación. La dimensión política y el concepto de pensamiento crítico en Freire y Giroux. Pedagogía y Saberes, (51), 133-144. doi: 10.17227/pys.num518286

(16) Ojeda, D., Villalobos, F. (2011). Elementos para una política pública desde la percepción del suicidio en Nariño. Revista colombiana de psicología, 20(1), 57-73.

(17) Organización Internacional para las Migraciones. (2015). Una paz estable, duradera y sensible a niños, niñas, adolescentes y jóvenes, Nariño. Bogotá, Colombia: Procesos digitales.

(18) Organización Internacional para las Migraciones. (2016). Aproximación al fenómeno de trata con fines de explotación sexual y laboral de niños, niñas y adolescentes en el departamento de Nariño. Bogotá, Colombia: Procesos digitales.

(19) Ospina, M., Mora, R. y Romero, J. (2013). Ecoturismo: diagnóstico y propuesta estratégica para la oferta de destinos ecoturísticos en Colombia por parte de las agencias de turismo localizadas en Bogotá, D.C. Cuadernos Latinoamericanos de Administración, 9(17), 7-28.

(20) Rodríguez, G. (2019). Economía ecológica: hacia una ecología en la enseñanza de la economía. Revista Kawsaypacha: sociedad y medio ambiente, (3), 89-104. doi: 10.18800/kawsaypacha.201901.004 
(21) Ruano, A. (2012). Desplazamiento forzado: modos de vida y relaciones sociales en la ciudad de Ipiales (Tesis de maestría). Universidad Nacional de Colombia.

(22) Santos, B. (2014). Descolonizar el saber / Reinventar el poder. Santiago de Chile: LOM.

(23) Santos, B. (2017). Justicia entre saberes. Epistemologías del Sur contra el Epistemicidio. Madrid, España: Morata.

(24) Santos, B. (2019). El fin del imperio cognitivo. Madrid, España: Trotta.

(25) Trouillot, M. (2011). Transformaciones globales. La antropología y el mundo moderno. Popayán, Colombia: Universidad del Cauca.

Cómo citar este artículo: Latif, S. y Niño, Y. (2020). Caracterización y motivaciones para el emprendimiento femenino en MIPYMES de Villavicencio - Colombia. Tendencias, 21(2), 167-190. https://doi.org/10.22267/rtend.202102.146 